\section{Immunohistochemical markers of neural progenitor cells in the early embryonic human cerebral cortex}

\author{
L. Vinci, ${ }^{1}$ A. Ravarino, ${ }^{1}$ V. Fanos, ${ }^{2}$ \\ A.G. Naccarato, ${ }^{3}$ G. Senes, ${ }^{1}$ C. Gerosa, ${ }^{1}$ \\ G. Bevilacqua, ${ }^{3}$ G. Faa, ${ }^{1}$ R. Ambu' ${ }^{1}$ \\ 'Department of Surgical Sciences, \\ Division of Pathology, University of \\ Cagliari \\ 2Department of Surgical Sciences, NICU \\ Center and Puericulture Institute and \\ Neonatal Section, University of Cagliari \\ ${ }^{3}$ Department of Pathology, Pisa \\ University Hospital, Italy
}

\section{Abstract}

The development of the human central nervous system represents a delicate moment of embryogenesis. The purpose of this study was to analyze the expression of multiple immunohistochemical markers in the stem/progenitor cells in the human cerebral cortex during the early phases of development. To this end, samples from cerebral cortex were obtained from 4 human embryos of 11 weeks of gestation. Each sample was formalin-fixed, paraffin embedded and immunostained with several markers including GFAP, WT1, Nestin, Vimentin, CD117, S100B, Sox2, PAX2, PAX5, T $\beta 4$, Neurofilament, CD44, CD133, Synaptophysin and Cyclin D1. Our study shows the ability of the different immunohistochemical markers to evidence different zones of the developing human cerebral cortex, allowing the identification of the multiple stages of differentiation of neuronal and glial precursors. Three important markers of radial glial cells are evidenced in this early gestational age: Vimentin, Nestin and WT1. Sox2 was expressed by the stem/progenitor cells of the ventricular zone, whereas the postmitotic neurons of the cortical plate were immunostained by PAX2 and NSE. Future studies are needed to test other important stem/progenitor cells markers and to better analyze differences in the immunohistochemical expression of these markers during gestation.

\section{Introduction}

The rapid and robust growth of the cerebral cortex, as compared to other brain areas, has been proposed as the crowning achievement of human evolution. Indeed, the cerebral cortex plays a critical role in cognitive functions, intelligence, language, motor abilities, memory and sensory perceptions that distinguish human beings from other animals species.

The mature human central nervous system (CNS) is composed by four major cell types: neurons, oligodendrocytes, astrocytes, and ependymal cells. All of these cell types are generated during embryogenesis from a common source, the neuroepithelial cells (NECs), that arise from the neural tube. This process, called neurogenesis, is characterized by cell proliferation, migration and differentiation and leads to the final creation of the 6-layered cortex. Cortical neurons fastly proliferate since the $10^{\text {th }}$ gestational week until the $22^{\text {nd }}$ week, then follows a phase of slower proliferation. ${ }^{1}$

In contrast to the neuroectodermal origin of the majority of CNS cells, microglia, the principal active immune defense cells of the brain, derives from the mesoderm. ${ }^{2}$ In recent years, microglia has been clarified to originate from two sources: the yolk sac and myeloid precursors. ${ }^{3}$ During the early stage of the neural tube development, NECs form the columnar monolayered epithelium that, at the end of neurulation, gives origin to the pseudostratified epithelium. The characteristic pseudostratification is mainly due to interkinetic nuclear migration. NECs undergo symmetric proliferative division leading to thickening of the neuroepithelium and growth of the neocortex. ${ }^{4}$ Cortical neurogenesis begins when NECs undergo asymmetric division: by self-renewing itself, one mother cells (NEC or apical radial glia) gives rise to one identical cell capable of self-renewal; the other daughter cell becomes either an apical intermediate progenitor, a basal progenitor cell or a newborn neuron. ${ }^{5}$ In the further stages of neurogenesis, NECs are progressively replaced by apical radial glia (aRG) cells that begins to express astroglial markers $^{6}$ and form radial fibers extending from their apical and basal poles. Radial glia cells play a key role during CNS development thank to their ability to generate neurons, astrocytes, oligodendrocytes and ependymal cells. Moreover, their fibers serve as a scaffold for neuronal migration from the ventricular zone toward definitive destination in the cortical plate. ${ }^{7}$ Like NECs, aRG cells undergo interkinetic nuclear migration in the ventricular zone. During neurogenesis progression, aRG cells switch from proliferation to differentiation. ${ }^{5}$ Apical intermediate progenitors, basal intermediate progenitors and basal radial glia can be generated either from NECs or aRG, and from themselves. ${ }^{4}$ Both types of basal progenitor cells are not attached to the ventricular surface and do not undergo interkinetic nuclear migration ${ }^{8}$. Accumulation of basal progenitor cells creates the subventricular zone, a distinct new germinal layer located above the
Correspondence: Laura Vinci, Division of Pathology, Department of Surgical Sciences, University of Cagliari, "S.Giovanni di Dio" Hospital, Via 0spedale 54, 09124 Cagliari, Italy. Tel. +39.070.6092372 - Fax: +39.070 .6092370 .

E-mail: lau.vinci@gmail.com

Key words: Cerebral cortex; human embryo; human development; immunohistochemistry; fetal stem cells.

Acknowledgments: the authors thank Prof Giacomo Cao (Dept. of Chemical and Materials Engineering, University of Cagliari) for relevant critical suggestions. Laura Vinci has performed her activity in the framework of the International $\mathrm{PhD}$ in Innovation Science and Technology at the University of Cagliari, Italy.

Contributions: RA LV GF AR VF AGN GB, provided conception and design of research; LV GS CG, performed experiments; RA LV GF AR CG, interpreted results of experiments; RA LV GF, drafted manuscript; RA GF VF AGN GB, conceived critical revision of the manuscript.

Conflict of interest: the authors declare that no conflict of interest exist.

Received for publication: 23 August 2015. Accepted for publication: 9 December 2015 .

This work is licensed under a Creative Commons Attribution-NonCommercial 4.0 International License (CC BY-NC 4.0).

\section{(C) Copyright L. Vinci et al., 2016}

Licensee PAGEPress, Italy

European Journal of Histochemistry 2016; 60:2563 doi:10.4081/ejh.2016.2563

ventricular zone. Apical intermediate progenitors maintain contact only with the ventricular surface (Figure 1). Apical progenitor cells in the ventricular zone and basal progenitor cells in the subventricular zone are generally considered to represent the major source of cortical neurons. ${ }^{1}$ Thanks to these processes, the embryonic cerebral cortex is layered into different regions: the ventricular zone, the subventricular zone, the intermediate zone, the subplate zone, the cortical plate, the pial zone (Figure 1). The differentiation of the neural progenitor cells is orchestrated by internal signals that are controlled by genes that carry information for all the structures and functions of a cell, and by external epigenetic signals including hormones and molecules secreted by other cells. Extrinsic factors, believed to be essential for maintenance and proliferation of the neural stem/progenitor cells pool, include Fibroblast Growth Factors (FGF), ${ }^{9}$ Epidermal Growth Factor (EGF), ${ }^{10}$ Sonic Hedgehog (SH), ${ }^{11}$ and Wnt family. ${ }^{12}$ Several immunohistochemical markers have 
been utilized in previous experimental studies for the identification of neural stem/progenitor cells in the developing cerebral cortex. Radial glia has been highlighted by several immunohistochemical markers including Vimentin, ${ }^{13}$ Nestin, ${ }^{14}$ S100B protein ${ }^{15}$ Pax6 ${ }^{16}$ and GFAP. ${ }^{17}$ Neural stem/progenitor cells have been reported to be reactive for Sox $2,{ }^{18}$ MSI-1 ${ }^{19}$ BMI- $1,{ }^{20}$ and Nestin. ${ }^{21}$ Moreover, a recent article from our group showed the expression of WT1 in the human brain. ${ }^{22} 0$ n the basis of these data, this work was aimed at studying the immunohistochemical phenotype of stem/progenitor cells in the human cerebral cortex during the early phases of development.

\section{Materials and Methods}

The expression of markers was evaluated in the frontal cerebral cortex from 4 human embryos from 11 weeks of gestation received from the Obstetric Division of the University of Cagliari, as voluntary termination of pregnancy (VTOP). All procedures performed were approved by the Ethics Human Studies Committee of University Medical Centre of Cagliari (according to the instructions of the Declaration of Helsinki). The frontal cerebral cortex of these fetuses has been sampled and histologically and immunohistochemically studied. Samples were fixed in $10 \%$ buffered formalin, routinely processed, and paraffin-embedded. Serial $3 \mu \mathrm{m}$-thick sections were obtained from each paraffin block; after dewaxing and rehydrating, one of these sections was stained with hematossilin-eosin, while the others were pretreated for immunohistochemical analysis, with

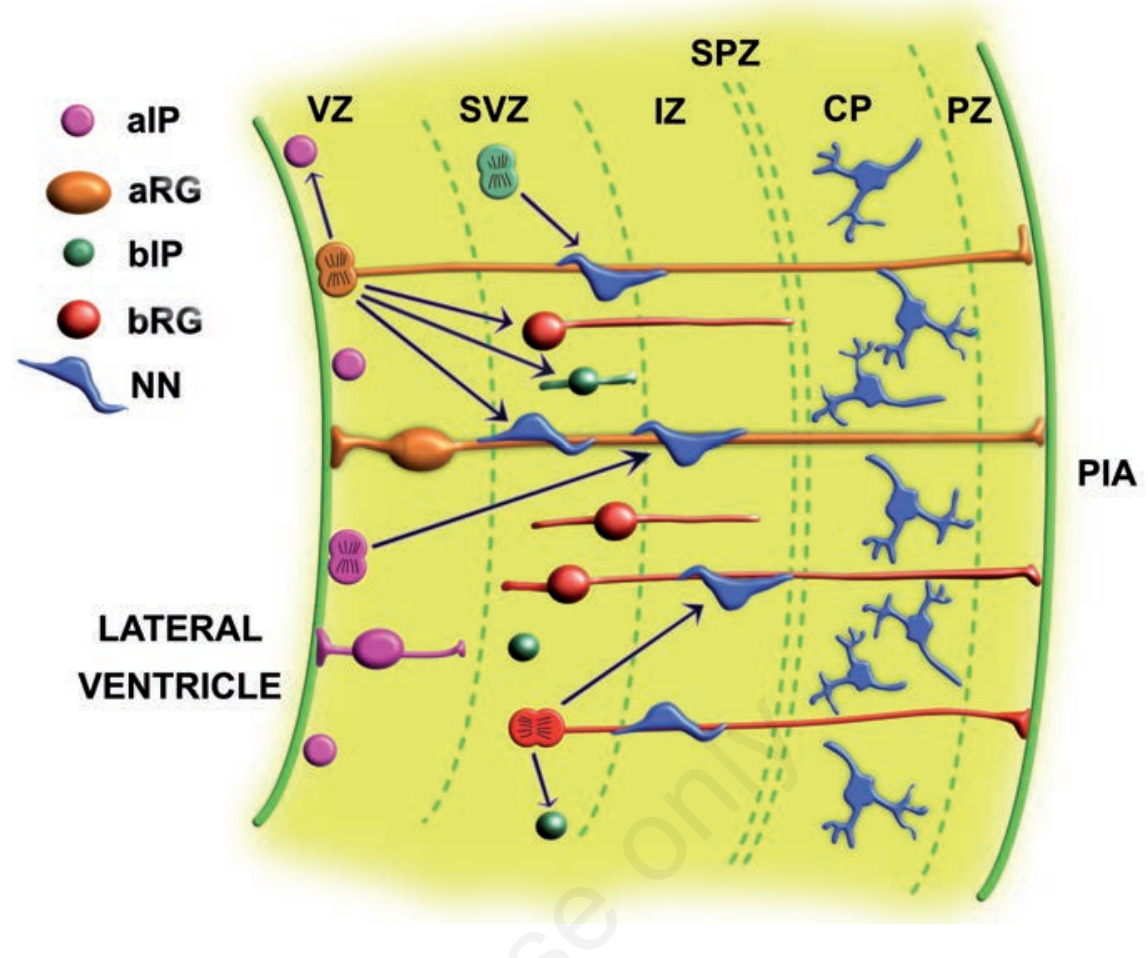

Figure 1. Schematic representation of cell types of the embryonic human cerebral cortex in the early stage of gestation. VZ, ventricular zone; SVZ, subventricular zone; IZ, intermediate zone; SPZ, subplate zone; CP, cortical plate; PZ, pial zone; aRG, apical radial glia; aIP, apical intermediate progenitor; bRG, basal radial glia; bIP, basal intermediate progenitor; NN, newborn neuron.

10 minutes heat-induced epitope retrieval (EnVision $^{\text {TM }}$ FLEX Target Retrieval Solution Dako Denmark A/S, Glostrup, Denmark - High pH Code: K8004; Low pH Code: K8005). Slides were then incubated for $20 \mathrm{~min}$ at room temper- ature with the antibodies reported in Table 1. Staining procedures were performed by Envision $^{\mathrm{TM}}$ FLEX+ (Dako, Code: K8002) Detection System and AutostainerLink 48 instrument following dealer's instructions.

Table 1. Antibodies utilized in this study.

\begin{tabular}{lcccc} 
Antibody & Dilution & Source & Company & \\
WT1 & $1: 100$ & Mouse monoclonal 6F-H2 & Dako & M3561 \\
Pax2 & $1: 400$ & Mouse monoclonal 3C7 & Abnova & H00005076-M01 \\
\hline S100B & $1: 2000$ & Rabbit polyclonal & Dako & Z0311 \\
Nestin & $1: 200$ & Mouse monoclonal 10C2 & Santa Cruz & SC-23927 \\
\hline Vimentin & $1: 500$ & Mouse monoclonal 3B4 & Dako & A4502 \\
CD117 & $1: 200$ & Rabbit polyclonal & Dako & SC-365823 \\
Sox2 & $1: 50$ & Mouse monoclonal E-4 & Santa Cruz & M0873 \\
NSE & $1: 200$ & Mouse monoclonal BBS/NCNI-H14 & Dako & SC-7297 \\
\hline CD44 & $1: 50$ & Mouse monoclonal DF1485 & Santa Cruz & GA65061 \\
PAX5 & $1: 30$ & Mouse monoclonal DAK-Pax5 & Dako & M0076 \\
Synaptophysin & $1: 20$ & Mouse monoclonal SY38 & Dako & M364201 \\
Cyclyn D1 & $1: 50$ & Rabbit monoclonal EP12 & Novocastra & NCL-GFAP-GA5 \\
GFAP & $1: 100$ & Rabbit polyclonal & Dako & M0762 \\
Neurofilament & $1: 50$ & Mouse monoclonal 2F11 & Abnova & PAB12663 \\
CD133 & $1: 200$ & Rabbit polyclonal & Bachem group & T4848 \\
Thymosyn $\beta 4$ & $1: 100$ & Rabbit polyclonal & &
\end{tabular}




\section{Results}

Among the markers tested in the embryonic human cerebral cortex in this study, we found immunoreactivity for WT1, Nestin, NSE, Pax2, Vim, CD117, S100B and Sox2. No reactivity was found for neurofilaments (NF), CD44, Thymosin $\beta 4 \quad$ (T 34$), \quad$ Pax5, GFAP, Synaptophysin, CD133 and Cyclin D1. Significant changes in reactivity for the different markers were observed between the ventricular zone, the intermediate zone and the cortical plate. The most important variations are reported in Figure 2.

\section{WT1}

At low power, immunoreactivity for WT1 appeared stronger in ventricular, subventricular and intermediate zones as compared to the cortical plate area. Immunostaining in the subventricular zone was mainly localized in the cytoplasmic projection of the radial glia extending from the ventricular zone toward the pial zone (Figure 3A). Nuclei of both ventricular neuroepithelial cells and radial glia did not showed reactivity for WT1. No immunostaining for WT1 was detected in the nuclei of cortical plate neurons, which appeared surrounded by a WT1-positive network formed by the cytoplasmic projection of radial glia cells (Figure 3B).

\section{Nestin}

Nestin-reactive cells are detected in the ventricular, subventricular and intermediate zones in the absence of a significant immunostaining in the cortical plate (Figure 4A). No immunostaining for Nestin was detected in the nuclei of the intermediate zone and the cortical plate cells. At higher magnification, immunostaing for Nestin was detected in the projection of radial glia cells extending from the ventricular zone toward the subplate zone (Figure 4B).

\section{Vimentin}

At a panoramic view, immunostaining for Vimentin was restricted to cytoplasmic projection of radial glia cells extending from the ventricular zone toward the subplate zone (Figure 5A). No immunostaining for Vimentin was detected in the other cells and in the nuclei of the radial glia cells (Figure 5B).

\section{PAX2}

At a panoramic view, PAX2 nuclear reactivity progressively increased from the ventricular zone toward the pial zone (Figure 6A). The cortical plate and the intermediate zone showed several cells with nuclear reactivity for PAX2 intermingled with no-reactive cells. At higher power, the vast majority of subpial zone cells showed a strong reactivity for PAX2 (Figure 6B). Pax2 scattered cells were also detected among pial cells (arrows).

\section{NSE}

At low magnification, immunostaining for NSE was detected in the intermediate zone, in the cortical plate and in the pial zone (Fig. 7A). No significant staining for NSE was observed in the ventricular neuroepithelium and in the subventricular zone. At higher magnification, the expression of NSE was detected in the cytoplasm of cells localized in the intermediate zone, in the absence of any significant reactivity in the subventricular area (Figure 7B).

\section{CD117}

At a panoramic view, CD117 immunostaining was mainly localized in the intermediate zone (Figure 8A). Moreover, a strong reactivity for CD117 was observed in a subpial strip (arrow). At higher power, CD117 reactivity in the intermediate zone appeared localized in the cellular cytoplasm (Figure 8B). Indeed, at this power a mild cytoplasmic immunoreactivity for CD117 was also detected in the cells of the cortical plate. No nuclear immunoreactivity for CD117 was found.

\section{S100B}

At low power, immunoreactivity for S100B was detected in the nuclei and cytoplasm of scattered cells mainly localized in the intermediate zone (Figure 9A). No significant staining was observed in the ventricular zone, in the subventricular zone and in the cortical plate. At higher power, S100B reactive cells appeared as large cells with thick cytoplasmic projection (Figure 9B).

\section{Sox2}

At panoramic view, immunostaining for Sox2 was particularly strong in both ventricular and subventricular zones, associated with a weaker immunostaining in the intermediate zone and in the cortical plate cells (Figure $10 \mathrm{~A})$. At higher magnification, immunostaining for Sox2 was detected in the nuclei of cells
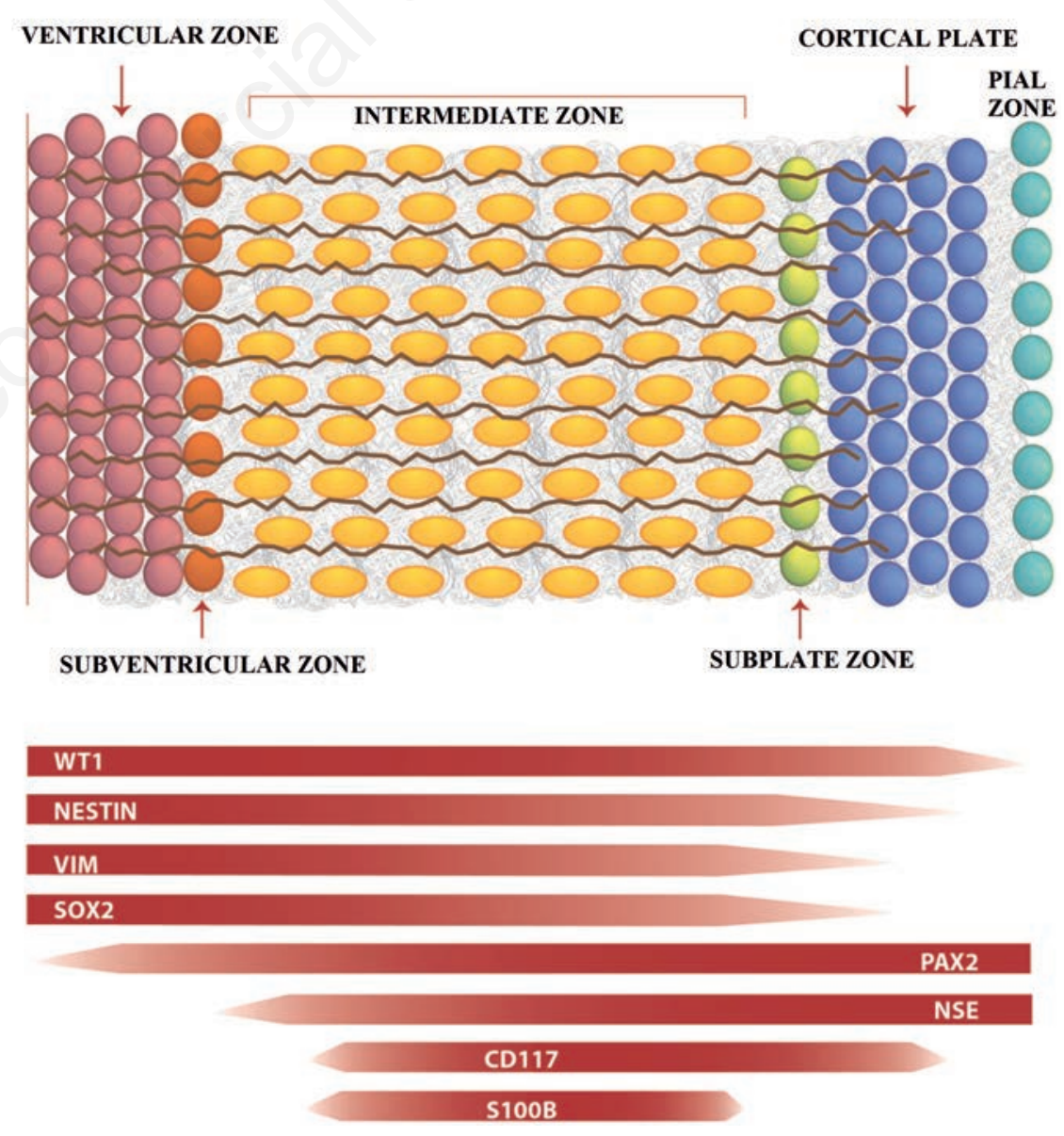

Figure 2. Trend of different expression of markers in different regions of developing cerebral cortex. 
localized in the ventricular zone, in the subventricular zone and in newborn neurons that were migrating in the intermediate zone toward the cortical plate (Figure 10B).

\section{Discussion}

The development of the human CNS represents a very delicate period of embryogenesis, being characterized by migration and differentiation of multiple cell types that may not be completely identified by morphology. Previous studies, mainly carried out on experimental models, evidenced that immunohistochemistry may allow the identification of different neural and glial precursors inside the ventricular, subventricular, intermediate, and the subplate zone and the cortical plate of the developing cortex. Our study confirms immunohistochemistry as a useful tool for the identification of precursor cells in the early developing human cortex. Indeed, immunoractivity for several cell markers allows the identification of different stages of differentiation of the neuronal and glial lineages. The most relevant finding of this study is the ability of immunohistochemistry to reveal the presence of radial glia cell bodies inside the ventricular zone and their projections along the whole cerebral cortex. Our findings evidenced three important markers of radial glial cells and neuronal precursors: Vimentin, Nestin and WT1. All these markers highlighted the function of radial glia cells that, thanks to their parallel long extensions, probably represent the most important guide for the radial migration of newborn neurons from the ventricular zone toward the pial zone. Among these radial glia markers, Vimentin and Nestin appeared the most specific ones, while WT1 was less selective, probably staining also other precursor cells.

Another interesting finding emerging from our study is the ability of the different immunohistochemical markers to immunostain the different zones of the developing cortex. The ventricular zone was intensely immunostained by WT1, Sox2, Nestin and Vimentin. The subventricular zone was evidenced by reactivity for PAX2, WT1 and Sox2. The intermediate zone was mainly immunoreactive for NSE and CD117; moreover, in this area $\mathrm{S} 100 \mathrm{~B}$ protein revealed the presence of scattered large cells, occasionally showing astrocitic-like appearance. The postmitotic neurons of cortical plate were immunostained by PAX2 and NSE; in this zone, interneural fibers were strongly reactive for WT1. The pial zone was stained by NSE, CD117 and WT1. Finally, scattered large cells in the pial zone showed immunoreactivity for PAX2. The majority of the immunohistochemical markers were
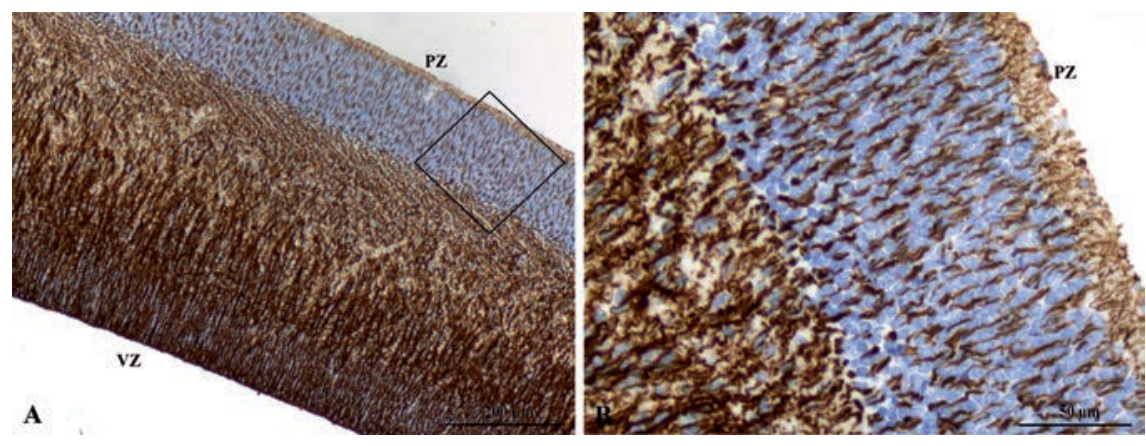

Figure 3. A) Immunoreactivity for WT1 in radial glia cells extending from the ventricular zone toward the pial zone. B) Nuclear negativity of cortical plate neurons surrounded by radial glia projections.
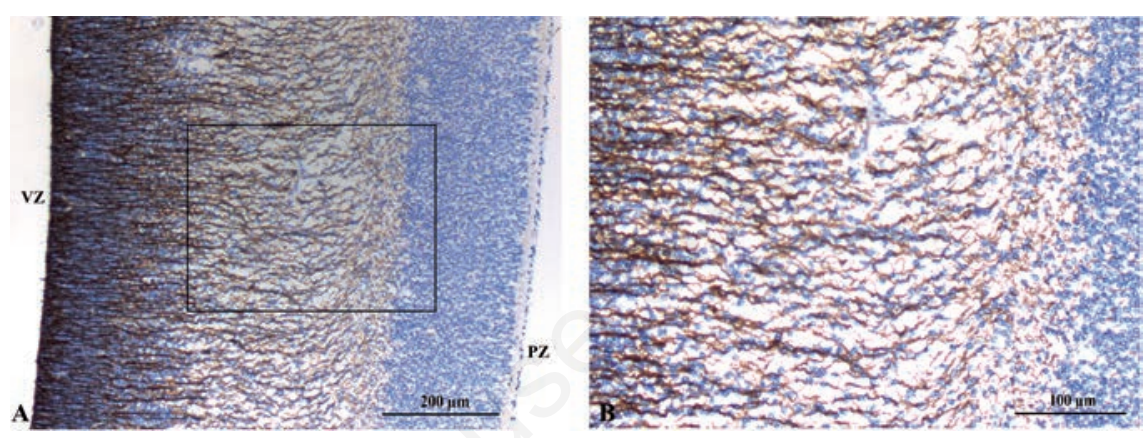

Figure 4. A) Nestin immunoreactivity in the ventricular, subventricular and intermediate zones. B) Positivity of radial glia cells extending from the ventricular zone toward the subplate zone.
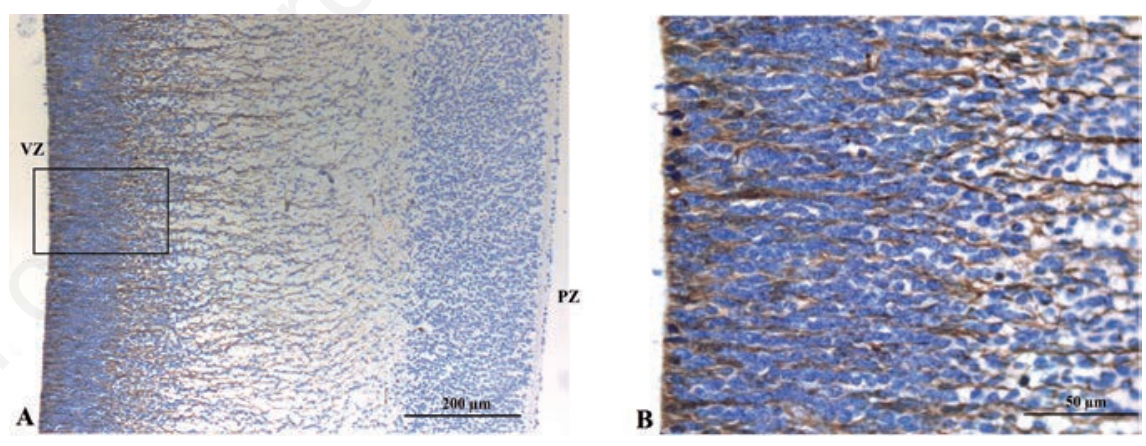

Figure 5. A) Vimentin immunoreactivity of radial glia cells extending from the ventricular zone toward the subplate zone. B) No reactivity of nuclei in both the ventricular and subventricular zones.
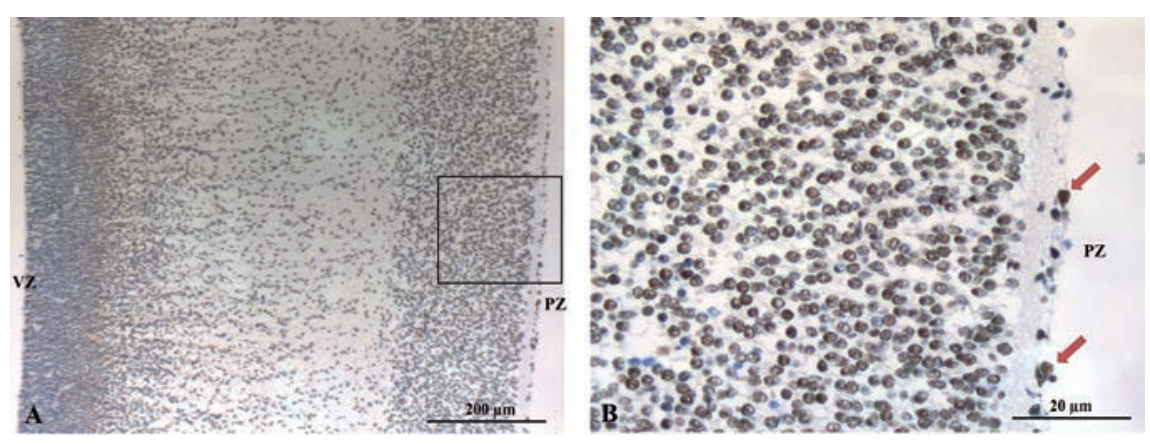

Figure 6. A) Pax 2 nuclear reactivity progressively increases from the ventricular zone toward the pial zone. B) Immunoreactivity of the cortical plate cells intermingled with no-reactive cells and scattered positive cells in the pial zone (arrows). 
localized in the cytoplasm of cell precursors, whereas immunoreactivity for PAX2 and Sox2 was restricted to the nuclei. S100B protein was localized both in the nuclei and cytoplasm in few scattered cells restricted in the intermediate zone of cerebral cortex.

The peculiar localization of the different immunohistochemical markers utilized in this study of the early developing human cerebral cortex deserves some consideration.

Sox 2, a member of the extended Sox family, ${ }^{23}$ is one of the earliest transcription factors expressed in the developing CNS. ${ }^{24}$ In the developing mouse neocortex, Sox 2 has been reported to be expressed in neural stem and progenitor cells. ${ }^{25}$ Our data confirm these previous experimental data, showing a prevalent Sox2 expression in the nuclei of the ventricular and subventricular zones, that represent the earliest precursor of the developing human cortex. PAX2 gene belongs to a family of genes that plays a critical role in the formation of multiple tissues and organs during embryonic development. ${ }^{26}$ Recently, PAX2 has been reported to be expressed during the formation of the central nervous system in human embryos. ${ }^{27}$ Our study allows to localize PAX2 expression in the precursor cells of subventricular zone, in the migrating neurons of the intermediate zone and in the postmitotic neurons in the cortical plate. These findings may suggest a possible role for $P A X 2$ in neuronal migration and differentiation. S100B is a protein of the S-100 protein family. ${ }^{28}$ In the cerebral cortex S100B protein has been reported to represent a glialspecific marker, being expressed primarily by astrocytes. ${ }^{29}$ Previous studies showed the expression of this protein in normal human fetal hippocampus, entorhinal cortex and occipital cortex. ${ }^{29} \mathrm{~S} 100 \mathrm{~B}$ reactivity in our study was restricted to scattered cells in the intermediate zone, whose morphology may be suggestive for their glial lineage. Nestin is an intermediate filament protein that has been reported to be expressed by the primitive neuroephitelium including developing astrocytes and neurons..$^{21}$ During neuro- and gliogenesis, Nestin is replaced by cell type-specific intermediate filaments such as NF in neurons and Glial fibrillary acidic protein (GFAP) in glial cells. ${ }^{31}$ In this study, Nestin immunostaining confirms these data, being mainly localized in radial glia bodies in the ventricular zone and in radial glia projections in the intermediate zone. Vimentin is a type III intermediate filament protein and acts as a crucial cytoskeletal component of mesenchymal cells, being involved in cell migration and in epithelial-tomesenchymal transition (EMT). ${ }^{32}$ Our data clearly indicate Vimentin as a good marker of radial glia cells in the absence of reactivity for that marker in the cortical plate, confirming the utility of Vimentin in the detection of early
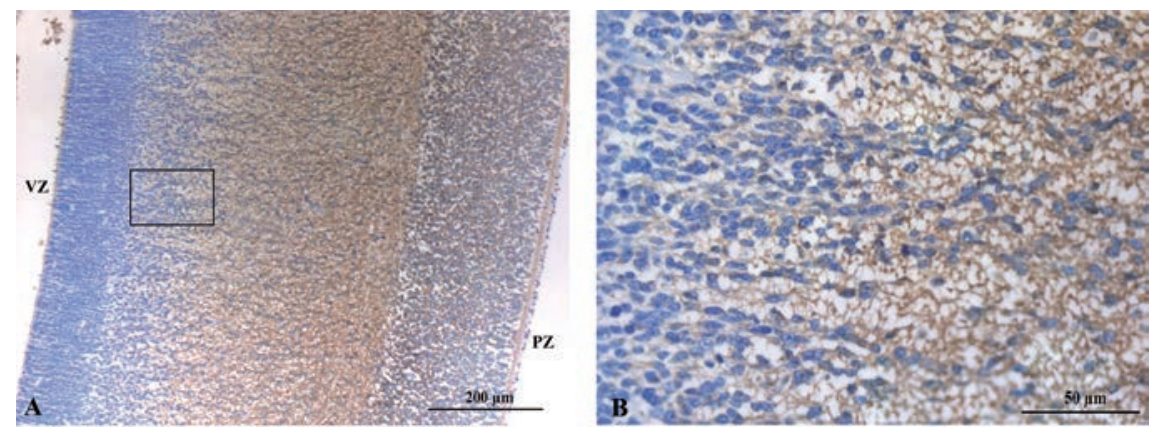

Figure 7. A) NSE immunostaining in the intermediate zone, in the cortical plate and in the pial zone. B) Cytoplasmic positivity in the intermediate zone cells in contrast to nonreactive cells of subventricular zone.
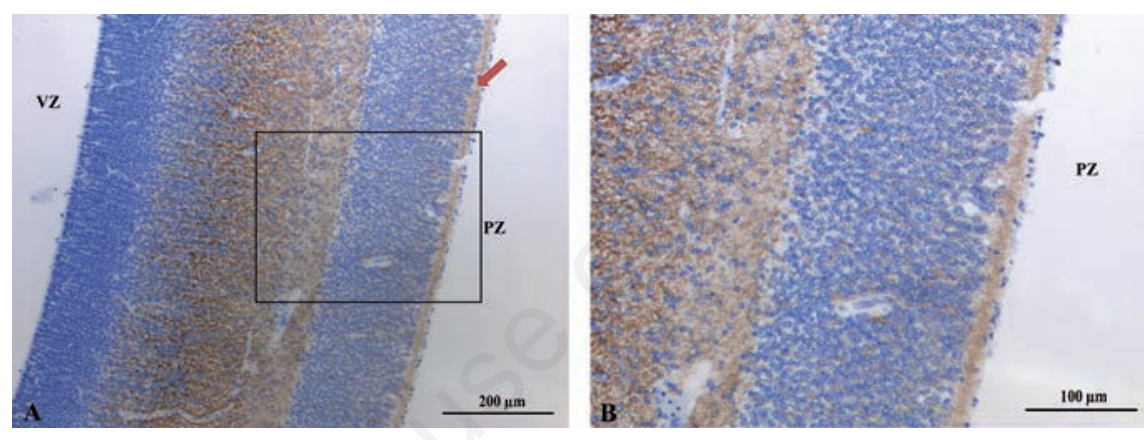

Figure 8. A) CD117 immunoreactivity in the intermediate zone and in subpial zone (arrow). B) Cytoplasmic positivity in the cortical plate.
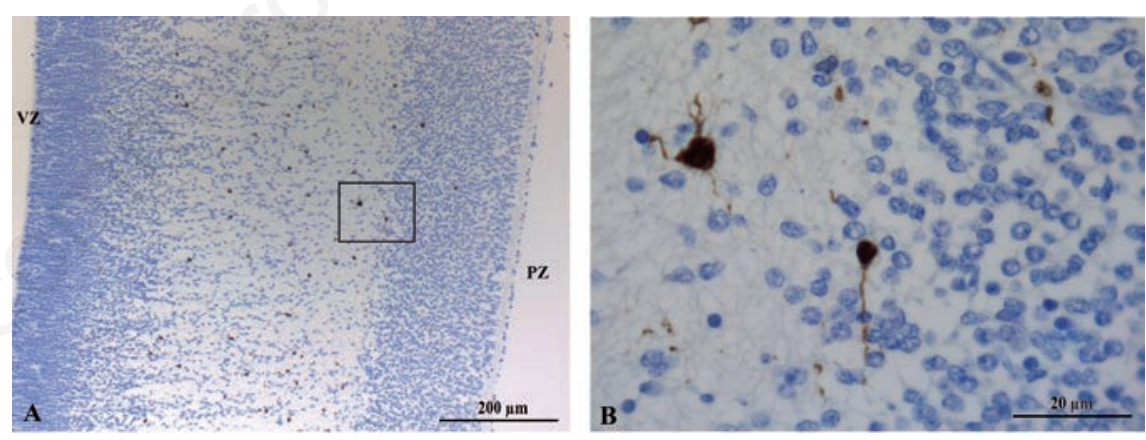

Figure 9. A) Nucler and cytoplasmic immunoreactivity for S100B in scattered cells localized in the intermediate zone. B) Higher magnification of the $\$ 100 \mathrm{~B}$ reactive cells in the intermediate zone
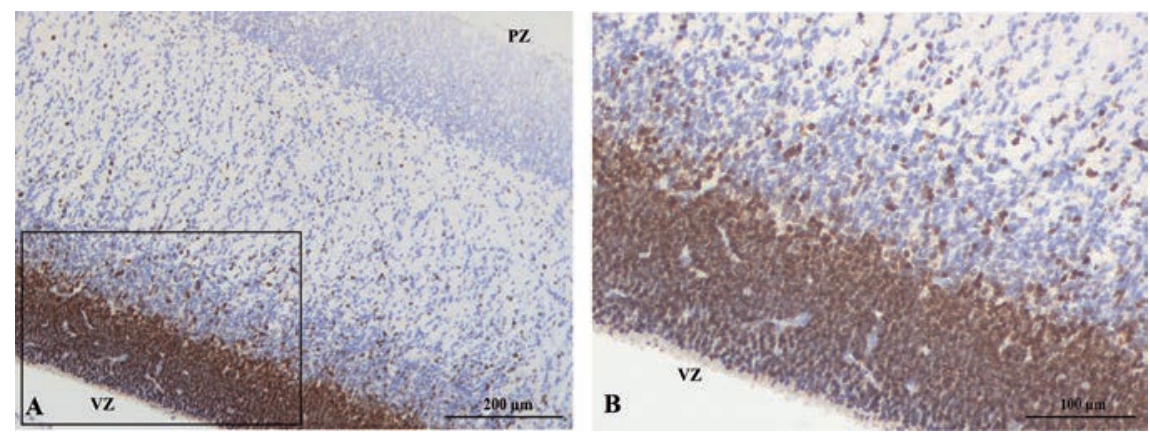

Figure 10. A) Sox 2 immunoreactivity decreases from the ventricular zone toward the cortical plate. B) Nuclear reactivity of cells localized in the ventricular zone, in the subventricular zone and in migrating cells. 
precursors in the developing human cerebral cortex. WT1 is a transcription factor highly expressed in several human organs during embryogenesis. $^{22}$ It has been suggested a potential role of WT1 protein in the development of the mouse retina, retinal ganglia ${ }^{33}$ and the olfactory system. ${ }^{34}$ Recently, a strong WT1 immunoractivity has been reported in the sympathetic system and in the gastroenteric nervous system of human fetuses..$^{35}$ In our study, WT1 was the marker with the strongest reactivity in all the cerebral cortex zones analyzed. In this early phase of human cerebral cortex development, the high expression of WT1 in all the cerebral cortex zones indicates a possible role for this protein in cells migration and differentiation. NSE is a glycolytic isoenzyme, which is expressed in central and peripheral neurons and in neuroendocrine cells. ${ }^{36}$ It is generally considered a marker of neural differentiation. ${ }^{37}$ In this study, the finding of a prevalent reactivity for NSE in the intermediate and subplate zones and in the cortical plate, associated with the absence of reactivity in the ventricular and subventricular zones, may indicate NSE as a marker of differentiation of the neuronal precursors in the human brain. $C D 117$ is the gene product of the c-kit proto-oncogene and act as a receptor for stem cell factor. Together with its ligand (SCF), it plays an important role in hematopoiesis. ${ }^{38} \mathrm{C}$ kit is a proto-oncogene involved in normal growth, development and neoplastic processes, and its product, CD117, is a highly specific immunohistochemical diagnostic marker for gastrointestinal stromal tumors (GISTs). In previous studies, CD117 has been reported to be expressed in central nervous system tumors.$^{39}$ Our study clearly evidences a role for CD117 in the development of human cerebral cortex. Its prevalent localization in the external layer of the intermediate zone and in the pial zone may suggest a role for this marker in the differentiation of neuronal cells and glial precursors. CD133 is considered a stem cell marker whose expression has been reported in human embryonic stem cells (hES) during their differentiation into neural cells, and in the ependymal cells of the mammalian postnatal forebrain. ${ }^{40-41}$ CD133 is also considered a neural cancer stem cells marker, ${ }^{42}$ being expressed in glioblastomas. ${ }^{43}$ In our study, we did not detected any reactivity for CD133 in the developing human cerebral cortex. The discrepancy between our and previous data may be due to the different models (animals and cell cultures) in which CD133 reactivity was previously reported.

Another important neural stem cell marker is CD44 which identifies astrocyte-restricted precursor cells. ${ }^{44}$ Previous animal models showed that this protein was expressed by progenitors of astrocytes and neurons in develop- ing cerebellum. ${ }^{45}$ In our study, no reactivity was found for CD44 in the fetal human cortex at 11 weeks of gestation. The absence of expression of NF, GFAP and Synaptophysin in our study reflects the lack of maturation of glial and neuronal precursors in the early phases of human gestation. In conclusion, our preliminary study shows the relevant role of immunohistochemistry in the detection of multiple stages of differentiation of neuronal and glial precursors in the early developing human cerebral cortex. Future studies are needed to detect differences in the immunohistochemical and mRNA expression patterns of stem/progenitor markers in the cerebral cortex at different gestational ages, in order to better evaluate the role of these markers in cell proliferation and differentiation during intrauterine human development.

\section{References}

1. Budday S, Steinmann P, Kuhl E. Physical biology of human brain development. Front Cell Neurosci 2015;9:257.

2. Ransohoff RM, Cardona AE. The myeloid cells of the central nervous system parenchyma. Nature 2010;468:253-62.

3. Ginhoux F, Greter M, Leboeuf M, Nandi S, See P, Gokhan S et al. Fate mapping analysis reveals that adult microglia derive from primitive macrophages. Science 2010;330: 841-5.

4. Florio M, Huttner WB. Neural progenitors, neurogenesis and the evolution of the neocortex. Development 2014;141:2182-94.

5. Götz M, Huttner WB. The cell biology of neurogenesis. Nat Rev Mol Cell Biol 2005;6:777-88.

6. Campbell K, Götz M. Radial glia: multi-purpose cells for vertebrate brain development. Trends Neurosci 2002;25:235-8.

7. Malatesta P, Appolloni I, Calzolari F. Radial glia and neural stem cells. Cell Tissue Res 2008;331:165-78.

8. Hansen DV, Lui JH, Parker PR, Kriegstein AR. Neurogenic radial glia in the outer subventricular zone of human neocortex. Nature 2010;464:554-61.

9. Gremo F, Presta M. Role of fibroblast growth factor-2 in human brain: a focus on development. Int J Dev Neurosci 2000;18: 271-9.

10. Garcez RC, Teixeira BL, Schmitt Sdos S, Alvarez-Silva M, Trentin AG. Epidermal growth factor (EGF) promotes the in vitro differentiation of neural crest cells to neurons and melanocytes. Cell Mol Neurobiol 2009;29:1087-91.

11. Ho KS, Scott MP. Sonic hedgehog in the nervous system: functions, modifications and mechanisms. Curr Opin Neurobiol 2002;12:57-63.

12. Kalani MY, Cheshier SH, Cord BJ, Bababeygy SR, Vogel H, Weissman IL, et al. Wnt-mediated self-renewal of neural stem/progenitor cells. Proc Natl Acad Sci USA 2008;105:16970-5.

13. Nakagawa T, Miyazaki T, Miyamoto 0 , Janjua NA, Hata T, Itano T. Regional expression of the radial glial marker vimentin at different stages of the kindling process. Epilepsy Res 2004;61:141-51.

14. Murdoch B, Roskams AJ. A novel embryonic nestin-expressing radial glia-like progenitor gives rise to zonally restricted olfactory and vomeronasal neurons. J Neurosci. 2008;28:4271-82.

15. Vives V, Alonso G, Solal AC, Joubert D, Legraverend C. Visualization of S100Bpositive neurons and glia in the central nervous system of EGFP transgenic mice. $\mathrm{J}$ Comp Neurol 2003;457:404-19.

16. Osumi N, Shinohara H, NumayamaTsuruta K, Maekawa M. Concise review: Pax6 transcription factor contributes to both embryonic and adult neurogenesis as a multifunctional regulator. Stem Cells 2008;26:1663-72.

17. Middeldorp J, Boer K, Sluijs JA, De Filippis L, Encha-Razavi F, Vescovi AL, et al. GFAPdelta in radial glia and subventricular zone progenitors in the developing human cortex. Development 2010;137:313-21.

18. Zhang S and Cui W. Sox2, a key factor in the regulation of pluripotency and neural differentiation. World J Stem Cells 2014;6: 305-11.

19. Kaneko J, Chiba C. Immunohistochemical analysis of Musashi-1 expression during retinal regeneration of adult newt. Neurosci Lett 2009;450:252-7.

20. Molofsky AV, Pardal R, Iwashita T, Park IK, Clarke MF, Morrison SJ. Bmi-1 dependence distinguishes neural stem cell selfrenewal from progenitor proliferation. Nature 2003;425:962-7.

21. Lendahl U, Zimmerman LB, McKay RD. CNS stem cells express a new class of intermediate filament protein. Cell 1990; 60:585-95.

22. Ambu R, Vinci L, Gerosa C, Fanni D, Obinu E, Faa A, et al. WT1 expression in the human fetus during development. Eur $\mathrm{J}$ Histochem 2015;59:2499.

23. Sasai Y. Roles of Sox factors in neural determination: conserved signaling in evolution? Int J Dev Biol 2001;45:321-6.

24. Avilion AA, Nicolis SK, Pevny LH, Perez L, Vivian N, Lovell-Badge R. Multipotent cell lineages in early mouse development depend on Sox2 function. Genes Dev 2003;17:126-40.

25. Bani-Yaghoub M, Tremblay RG, Lei JX, 
Zhang D, Zurakowski B, Sandhu JK, et al. Role of Sox2 in the development of the mouse neocortex. Dev Biol 2006;295:5266.

26. Terzić J, Muller C, Gajović S, Saraga-Babić $M$. Expression of PAX2 gene during human development. Int J Dev Biol 1998; 42:701-7.

27. Namm A, Arend A, Aunapuu M. Expression of Pax2 protein during the formation of the central nervous system in human embryos. Folia Morphol (Warsz) 2014; 73:272-8.

28. Zimmer DB, Cornwall EH, Landar A, Song W. The S100 protein family: history, function, and expression. Brain Res Bull 1995;37:417-29.

29. Adami C, Sorci G, Blasi E, Agneletti AL, Bistoni F, Donato R. S100B expression in and effects on microglia. Glia 2001;33:131-42.

30. Tiu SC, Chan WY, Heizmann CW, Schäfer BW, Shu SY, Yew DT. Differential expression of $\mathrm{S} 100 \mathrm{~B}$ and $\mathrm{S} 100 \mathrm{~A} 6(1)$ in the human fetal and aged cerebral cortex. Brain Res Dev Brain Res 2000;119:159-68.

31. Halliday GM, Cullen KM, Kril JJ, Harding AJ, Harasty J. Glial fibrillary acidic protein (GFAP) immunohistochemistry in human cortex: a quantitative study using different antisera. Neurosci Lett 1996;209:29-32.

32. Ivaska J. Vimentin: Central hub in EMT induction? Small GTPases 2011;2:51-3.
33. Wagner KD, Wagner N, Vidal VP, Schley G, Wilhelm D, Schedl A, et al. The Wilms' tumor gene Wt1 is required for normal development of the retina. EMBO J 2002;21:1398-405.

34. Wagner N, Wagner KD, Hammes A, Kirschner KM, Vidal VP, Schedl A, et al. A splice variant of the Wilms' tumour suppressor WT1 is required for normal development of the olfactory system. Development 2005;132:1327-36.

35. Parenti R, Puzzo L, Vecchio GM, Gravina L, Salvatorelli L, Musumeci G, et al. Immunolocalization of Wilms' Tumor protein (WT1) in developing human peripheral sympathetic and gastroenteric nervous system. Acta Histochem 2014;116:48-54.

36. Marangos PJ, Schmechel DE, Parma AM, Goodwin FK. Developmental profile of neuron-specific (NSE) and non-neuronal (NNE) enolase. Brain Res 1980;190:185-93.

37. Mareschi K, Novara M, Rustichelli D, Ferrero I, Guido D, Carbone E et al. Neural differentiation of human mesenchymal stem cells: Evidence for expression of neural markers and eag $\mathrm{K}+$ channel types. Exp Hematol 2006;34:1563-72.

38. Sperling C, Schwartz S, Büchner T, Thiel E, Ludwig WD. Expression of the stem cell factor receptor C-KIT (CD117) in acute leukemias. Haematologica 1997;82:617-21.
39. Cetin N, Dienel G, Gokden M. CD117 expression in glial tumors. J Neurooncol 2005;75:195-202.

40. Peh GS, Lang RJ, Pera MF, Hawes SM. CD133 expression by neural progenitors derived from human embryonic stem cells and its use for their prospective isolation. Stem Cells Dev 2009;18:269-82.

41. Coskun V, Wu H, Blanchi B, Tsao S, Kim K, Zhao J, et al. CD133+ neural stem cells in the ependyma of mammalian postnatal forebrain. Proc Natl Acad Sci USA 2008; 105:1026-31.

42. Sykes AM, Huttner WB. Prominin-1 (CD133) and the cell biology of neural progenitors and their progeny. Adv Exp Med Biol 2013;777:89-98.

43. Sanai N, Alvarez-Buylla A, Berger MS. Neural stem cells and the origin of gliomas. N Engl J Med 2005;353:811-22.

44. Liu Y, Han SS, Wu Y, Tuohy TM, Xue H, Cai $\mathrm{J}$ et al. CD44 expression identifies astrocyte-restricted precursor cells. Dev Biol 2004;276:31-46.

45. Naruse M, Shibasaki K, Yokoyama S, Kurachi M, Ishizaki Y. Dynamic changes of CD44 expression from progenitors to subpopulations of astrocytes and neurons in developing cerebellum. PLoS One 2013; 8:e53109. 